\title{
Tracheobronchoplasty for tracheomalacia
}

\author{
Cameron D. Wright, Douglas J. Mathisen \\ Division of Thoracic Surgery, Massachusetts General Hospital, Boston, MA, USA \\ Correspondence to: Cameron D. Wright, MD. Division of Thoracic Surgery, Founders 7, Massachusetts General Hospital, 55 Fruit Street, Boston, MA \\ 02114, USA. Email: cdwright@mgh.harvard.edu.
}

\begin{abstract}
Tracheobronchomalacia is an uncommon acquired disorder of the central airways. Common symptoms include dyspnea, constant coughing, inability to raise secretions and recurrent respiratory infections. Evaluation includes an inspiratory-expiratory chest computed tomography (dynamic CT), an awake functional bronchoscopy and pulmonary function studies. Patients with significant associated symptoms and severe collapse on CT and bronchoscopy are offered membraneous wall plication. Tracheobronchoplasty is performed through a right thoracotomy. The posterior airway is exposed after the azygous vein is ligated. The posterior wall of the trachea (and usually both main bronchi) is plicated to a sheet of thick acellular dermis (or polypropylene mesh) with a series of 4 mattress sutures of 4-0 sutures from the thoracic inlet to the bottom of the trachea to re-shape the trachea and restore the normal D shape. Patients report generally good results with improvement of their symptoms. Quality of life is usually improved while pulmonary function tests usually are not improved.
\end{abstract}

Keywords: Tracheoplasty; tracheomalacia; tracheal surgery

Submitted Nov 06, 2017. Accepted for publication Jan 08, 2018.

doi: 10.21037/acs.2018.01.19

View this article at: http://dx.doi.org/10.21037/acs.2018.01.19

\section{Introduction}

Tracheobronchomalacia in adults is uncommon and often an incidental finding on a chest CT. Severe airway collapse $(>90 \%)$ is rare and usually associated with symptoms such as dyspnea, incessant coughing, inability (or difficulty) raising secretions and repeated chest infections (1). These patients are often miserable and there is often a delay in diagnosis as many clinicians are not familiar with this uncommon disease. If patients are smokers and have associated chronic obstructive pulmonary disease (COPD) it can be very problematic and challenging to sort out which disease is driving the patient's symptoms and interfering with their quality of life. In general it is best to refrain from operating on those with significant COPD unless one is quite convinced that the tracheobronchomalacia is very dominant. The evaluation process starts with a high quality dynamic chest CT with inspiratory and expiratory views to determine the degree of tracheobronchomalacia, whether it extends to the major bronchi, and to screen for other lung diseases (Figure 1). If severe malacia is present then an awake functional bronchoscopy is done to verify this and further assess the airway. All airways collapse with a strong cough and that finding is not diagnostic of tracheobronchomalacia. During quiet breathing with exhalation, if the airway collapses $90 \%$ or more then more severe malacia is confirmed and a diagnosis of tracheomalacia is valid. Sometimes coughing will be incited by the opposition of the anterior and posterior walls of the airway. There are two anatomic forms of tracheomalacia, the classic soft/ weak anterior tracheal cartilages (cartilaginous malacia) with a redundant posterior membranous wall (can be seen with tracheobronchomegaly-Mounier Kuhn syndrome) and excessive forward displacement of the membranous wall [membranous malacia, excessive dynamic airway collapse (EDAC)]. Caution must be used in evaluating patients with morbid obesity as most will have at least moderate EDAC and it is unclear how these patients fare with tracheoplasty. Patients with morbid obesity have other reasons for dyspnea including a restrictive component, deconditioning and pulmonary hypertension from obstructive sleep apnea. Pulmonary function tests are performed to document 

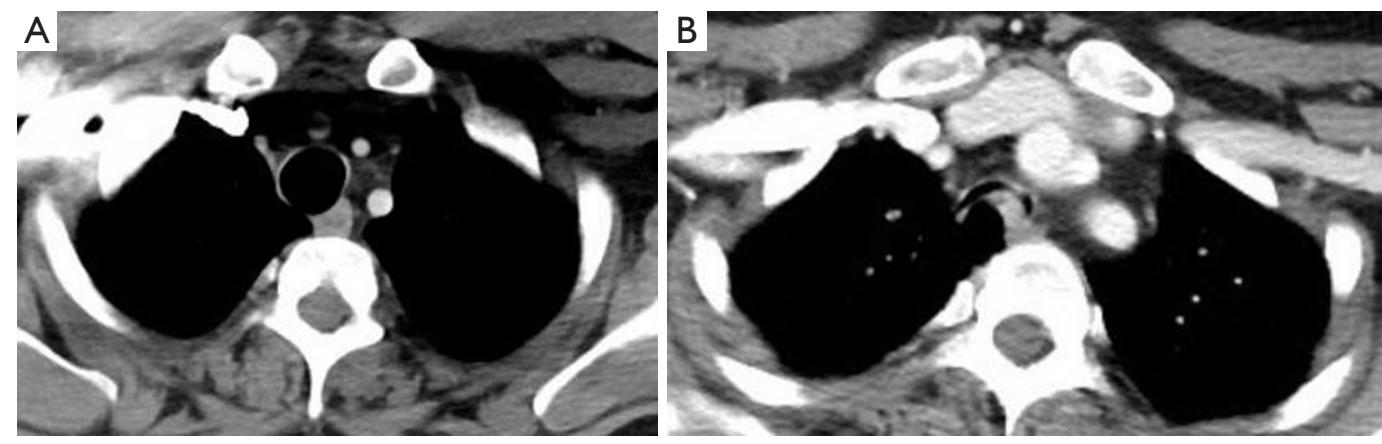

Figure 1 CT scans are helpful to screen for tracheomalacia. (A) Axial CT scan of a patient during inspiration with severe tracheomalacia; (B) axial CT scan of the same patient during expiration. Notice the almost complete collapse of the tracheal lumen.

any other lung pathology and help assess the risk for thoracotomy. Lung function tests are often relatively normal in patients with tracheomalacia in the absence of any other pulmonary disease. There is no specific finding on pulmonary function studies that is diagnostic of tracheobronchomalacia. Some authors have advocated a short trial of a silicone $\mathrm{Y}$ stent or a self-expanding metallic stent in an attempt to document whether symptoms and quality of life are improved with airway stenting as a diagnostic strategy to decide if tracheobronchoplasty would be beneficial $(2,3)$. However temporary stenting is not always helpful as many patients have not been able to tolerate a stent even for 1 day due to coughing and airway irritation. In addition airway infections and mucus plugging of the stent are not uncommon complications of a temporary stent. In general an operation is recommended if severe symptoms match the imaging and bronchoscopic findings of severe expiratory collapse of the airway.

The principle of membranous wall splinting is to stabilize and add rigidity in the case of membranous malacia and to also reconfigure the normal shape of the trachea in the case of cartilaginous malacia (Figure 2). Cartilaginous malacia is characterized by a widened trachea with a redundant membranous wall. Patients with membranous malacia usually have a trachea of normal size (approximately $2.5 \mathrm{~cm}$ from side to side in the adult) and thus the membranous wall is not redundant, just hyper-mobile. The posterior membranous wall of the trachea is reinforced with sequential rows of mattress 4-0 sutures placed in a partial thickness fashion. Typically, four sutures are placed across the membranous wall with the lateral sutures also catching a small bite of the lateral cartilaginous wall of the trachea. The surgeon needs to estimate the degree of reduction in the width of the membranous wall that will recreate the $\mathrm{D}$ shape of the trachea. This can be done by pinching the middle of the membranous wall together and assessing the shape of the cartilaginous trachea until the desired shape and size are achieved. The distance between the lateral walls of the trachea is then measured and used to cut the appropriate size of the stabilizing material. Our group first used polypropylene mesh for membranous wall stabilization, reasoning it was easy to use, flexible but did not stretch and resisted infection. Most surgeons continue to use it. I no longer use it as I have had two patients develop late erosion of the mesh into the airway which created a very challenging late complication with severe granulation tissue and infection in the airway. I currently use extra thick acellular dermis with good medium term results. We tried polytetrafluoroethylene (PTFE) once but the patient developed a fluid collection between the PTFE sheet and the membranous wall that caused obstruction of the airway. Whatever is used must be safe to provide stabilization of the airway for decades but not erode into either the esophagus or airway. Different sutures have been used for fixing the splinting material to the membranous wall. These include polypropylene, polyglactin 910 and polydioxanone. It is not clear what is the best suture material to use, permanent or temporary. It is not uncommon when attempting to place partial thickness sutures that they are actually full thickness into the airway, thus potentially introducing bacteria next to the splint and a foreign body into the airway. Polydioxanone eventually dissolves thus eliminating the foreign body in the airway issue but potentially would allow the repair to weaken and fall apart with time. Currently polydioxanone is used with good results.

\section{Operation}

All patients have a thoracic epidural placed for postoperative 


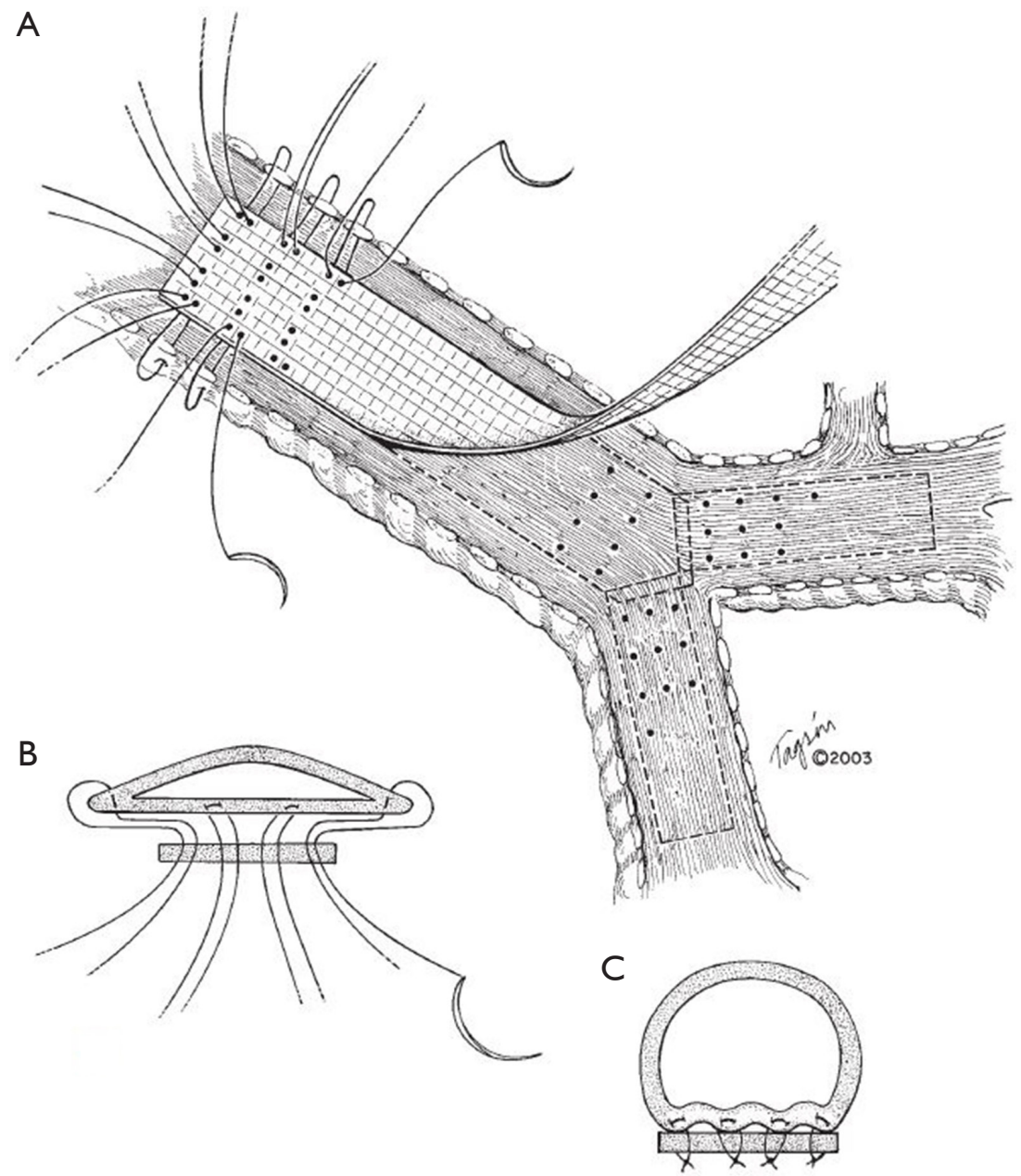

Figure 2 Schematic drawing of the key elements of a posterior wall membraneous wall tracheoplasty. (A) The membranous wall splinting material is carefully sutured with partial thickness fine 4-0 sutures to the posterior membranous wall to stiffen the membranous wall and reconfigure the shape of the trachea. The strip of the splinting material (I currently use extra thick Alloderm) is carefully measured such that when the sutures are tied the trachea is reconfigured to a more normal shape. Typically 4 rows of sutures are placed across the trachea while 3 are placed across the main bronchi; (B) cross-sectional diagram showing spacing of sutures. The lateral ones restore the D curve of the trachea and the central ones fix the redundant membranous wall to the splinting material. The sutures ideally do not penetrate the tracheal lumen; (C) the completed tracheoplasty with restoration of the normal shape of the trachea.

pain control. General anesthesia is used and the patient is intubated with an extra-long wire reinforced single lumen endotracheal tube that is positioned with the aid of a bronchoscope in the distal left main bronchus. A double lumen tube is not used as its large size can interfere with suturing the membranous wall. The patient is placed in the standard left lateral decubitus position and padded appropriately. A high standard right posterolateral thoracotomy is performed in the $4^{\text {th }}$ interspace. The azygous vein is divided and the mediastinal pleura is incised over the membranous wall of the trachea and main bronchi. The vagus nerve is preserved. The posterior airway is fully exposed from the thoracic inlet to the main bronchi. The lateral and anterior aspects of the trachea are not dissected out to avoid damage to the recurrent laryngeal nerves and to avoid devascularizing the trachea. If the main bronchi are involved then the dissection extends down to the right bronchus intermedius and the down the left main bronchus to the upper lobe takeoff.

Once the posterior airway is exposed the splinting 
material must be cut and fashioned to the appropriate width and length (Figure 2). If the main bronchi need to be splinted as well either separate strips can be cut for each main bronchus (usually $1 \mathrm{~cm}$ for the left main bronchus and $1.5 \mathrm{~cm}$ for the right main bronchus) or a $\mathrm{Y}$ shaped strip can be cut out of wider material so there is one continuous strip. Cutting the splinting material as a $\mathrm{Y}$ appears somewhat easier and is currently the preferred method. The repair is started by anchoring the distal tracheal end at the carina with a stitch at the carinal spur and in the middle of the main bronchi and at the lateral edges of the tracheobronchial angle. Middle sutures are placed in a mattress fashion thru the splint, then a partial thickness bite thru the membranous wall and then back thru the splint again. These are placed one-third and two thirds of the way across the membranous wall. The lateral stitches catch the junction of the membranous wall and the cartilaginous wall for extra strength. All four stitches are placed across one row, each individually snapped and then organized on the drapes and are tied after the next row of sutures are placed. The next row of four sutures are placed and then the preceding row is tied. It is easier to place the stiches and position the splint for optimal suturing if the preceding row is not tied down. Each row is placed about $5-7 \mathrm{~mm}$ apart and the repair is continued until the thoracic inlet area is reached. The malacia almost always stops at the thoracic inlet so there is usually no need to try and expose the trachea in the neck. Once the trachea is done the bronchi are done next if required. The right side is done first and then the left. The left is the most difficult due to poor exposure deep in the mediastinum and the presence of the endotracheal tube. The cuff should be temporarily deflated and ventilation stopped when suturing the left main bronchus so the cuff is not inadvertently punctured.

If possible sutures should be partial thickness in the airway. However practically speaking it is not uncommon to find at the end during surveillance bronchoscopy that there are several (out of dozens) inadvertent sites of suture penetration. It is not necessary to go back and remove those and replace them as it would be very challenging to identify each full-thickness suture.

Surveillance bronchoscopy at the conclusion of the procedure is done by pulling the endotracheal tube back to the proximal trachea and inspecting the result of the repair during inspiration and expiration. Any significant residual pathology or inadvertent issues (such as narrowing of the right upper lobe bronchus if the main and intermedius is splinted) should be identified and corrected at this time rather than postoperatively. A single chest tube is placed and the thoracotomy is closed in the routine fashion. Patients are extubated in the operating room and often require some temporary positive pressure support until they are fully awake. Observation in the ICU is usually advised as these patients often have airway issues and difficulty clearing secretions for a few days. Care is otherwise routine as for a major thoracotomy.

\section{Discussion}

Patients who are properly selected and have a good anatomic result often mention early in the postoperative period they feel that they are better despite the presence of a painful thoracotomy incision (4-6). The morbidity is usually pulmonary in nature and runs the gamut of atelectasis, sputum retention, pneumonia and respiratory failure. Mortality after operation is quite rare. Quality of life measures are improved in most patients and functional testing with the 6 -minute walk test also demonstrates improvement in most patients $(5,6)$. Pulmonary function tests usually do not measurably improve. Certainly one of the problems in evaluating these patients (either preoperatively or postoperatively) is the subjective nature of their symptoms and how they perceive their respiratory health. In the end the goal is a satisfied patient with an improved quality of life despite the lack of a quantifiable metric. Indeed postoperative surveys of patients have reported that most patients are improved and satisfied with the results of a tracheoplasty (4-7). Rarely patients may return with symptoms of tracheomalacia again after operation and may have isolated cervical tracheomalacia. Cervical tracheoplasty has been reported to improve patients with that unusual condition (8). Although most reports use a thoracotomy approach, a video-assisted thoracic surgery (VATS) approach has been reported as well (9). A recent report on a patient with post-tuberculosis tracheomalacia used 3-D printing techniques to create an external scaffold to suspend and thus enlarge the tracheal lumen (10).

\section{Acknowledgements}

None.

\section{Footnote}

Conflicts of Interest: The authors have no conflicts of interest 
to declare.

\section{References}

1. Wright CD, Grillo HC, Hammoud ZT, et al. Tracheoplasty for expiratory collapse of central airways. Ann Thorac Surg 2005;80:259-66.

2. Murgu SD, Colt HG. Tracheobronchomalacia and excessive dynamic airway collapse. Respirology 2006;11:388-406.

3. Ernst A, Majid A, Feller-Kopman D, et al. Airway stabilization with silicone stents for treating adult tracheobronchomalacia: a prospective observational study. Chest 2007;132:609-16.

4. Majid A, Alape D, Kheir F, et al P. Short-Term Use of Uncovered Self-Expanding Metallic Airway Stents for Severe Expiratory Central Airway Collapse. Respiration 2016;92:389-96.

5. Wright CD, Grillo HC, Hammoud ZT, et al. Tracheoplasty for expiratory collapse of central airways.

Cite this article as: Wright CD, Mathisen DJ. Tracheobronchoplasty for tracheomalacia. Ann Cardiothorac Surg 2018;7(2):261-265. doi: 10.21037/acs.2018.01.19
Ann Thorac Surg 2005;80:259-66.

6. Gangadharan SP, Bakhos CT, Majid A, et al. Technical aspects and outcomes of tracheobronchoplasty for severe tracheobronchomalacia. Ann Thorac Surg 2011;91:157480; discussion 1580-1.

7. Buitrago DH, Wilson JL, Parikh M, et al. Current concepts in severe adult tracheobronchomalacia: evaluation and treatment. J Thorac Dis 2017;9:E57-E66.

8. Wilson JL, Folch E, Kent MS, et al. Posterior Mesh Tracheoplasty for Cervical Tracheomalacia: A Novel Trachea-Preserving Technique. Ann Thorac Surg 2016;101:372-4.

9. Tse DG, Han SM, Charuworn B, et al. Video-assisted thoracoscopic surgical tracheobronchoplasty for tracheobronchomalacia. J Thorac Cardiovasc Surg 2011;142:714-6.

10. Huang L, Wang L, He J, et al. Tracheal suspension by using 3-dimensional printed personalized scaffold in a patient with tracheomalacia. J Thorac Dis 2016;8:3323-8. 